\title{
Cerebral aneurysms one year after resection of a cardiac myxoma
}

\author{
A. W. G. J. Oomen • S. H. H. Kuijpers
}

Published online: 20 April 2013

(C) The Author(s) 2013. This article is published with open access at Springerlink.com

\section{Case}

A 40-year-old Caucasian woman was referred to the outpatient clinic of the cardiology department for analysis of a possible cardiac embolus. She was dyspnoeic on exertion, functional class NYHA III/IV and had complaints of malaise and fever. Earlier she had been analysed by internal medicine for the fever and malaise. No cause was found, but on CT abdomen an infarction of the spleen and liver was seen.

A transthoracic echocardiogram showed a large mass in the left atrium with obstruction of the mitral valve and pulmonary hypertension (Fig. 1). The diagnosis of cardiac myxoma was made and the patient was referred for resection of the mass. This proceeded without complications and pathological analysis confirmed the diagnosis of myxoma.

Soon after this, her complaints resolved completely and the laboratory results returned to normal. A follow-up transthoracic echocardiogram showed no indications of a reoccurrence.

One year later she presented to the emergency department with complaints of sensory loss in her tongue and face, and word finding difficulty. These complaints had existed for 1 week and had already partly resolved. Neurological examination was without further abnormalities. A CT scan showed six haemorrhagic lesions in the right cerebral hemisphere. A following MRI scan of the brain also showed multiple haemorrhagic lesions and was suggestive for cerebral micro-aneurysms (Fig. 2). A transthoracic echocardiogram showed no signs of recurrence of the myxoma.

\footnotetext{
A. W. G. J. Oomen $(\bowtie)$

Department of Cardiology, Catharina Hospital,

Michelangelolaan 2, 5623 EJ Eindhoven,

the Netherlands

e-mail: adoomenjr@gmail.com

S. H. H. Kuijpers

Department of Cardiology, Maxima Medisch Centrum,

De Run 4600, 5504 DB Veldhoven, the Netherlands
}

There were also no indications of vasculitis. Cerebral angiography showed micro-aneurysms in the right middle cerebral artery (Fig. 3). The diagnosis myxoma-related cerebral micro-aneurysms was made. These findings and the management options were discussed with the department of neurosurgery and it was decided to treat her conservatively. Up till now she is doing relatively well. Her complaints have largely resolved. She is being treated medically for epileptic seizures and occasionally she suffers from headaches and tingling of her tongue. A follow-up cerebral MRI showed no progression of the lesions.

\section{Discussion}

Primary tumours of the heart are rare. In recent issues of this journal two cases were published $[1,2]$. The incidence was reported between 0.0017 and $0.19 \%$ in an unselected population at autopsy [3]. About three-quarters of these tumours are benign and half of them are myxomas. Cardiac myxomas arise in the left atrium in about $75 \%$ of cases. The majority of the others originate in the right atrium and sporadically in the left and right ventricle [4].

Patients with cardiac myxoma usually present with symptoms of cardiac obstruction, constitutional symptoms such as fever, weight loss or malaise or neurological symptoms due to embolisation. Up to $45 \%$ of cardiac myxomas are associated with systemic embolism, with involvement of the brain in half of the cases [5]. In 2010, Liesting et al. described in this journal a case of systemic embolisation as presentation of recurrence of a cardiac myxoma 2 years after surgery [6].

However, delayed aneurysm formation after resection of cardiac myxoma is rare. Only a few case reports are documented [7-14].

The pathogenesis of formation of myxoma-related aneurysms is not clear with two hypotheses accepted in the literature. The original hypothesis was that temporary occlusion of 


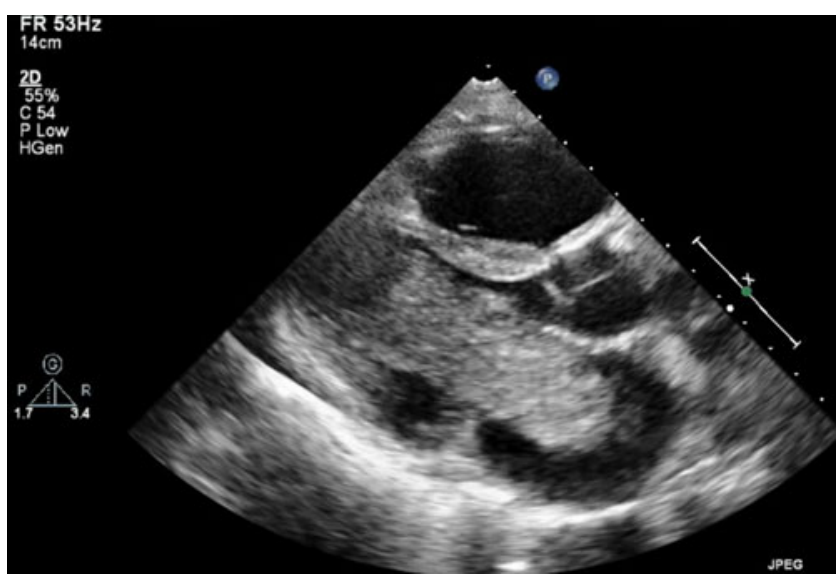

Fig. 1 Transthoracic echocardiogram, parasternal long axis, showing a large mass in the left atrium protruding through the mitral valve into the left ventricle during diastole

cerebral vessels by tumour emboli leads to endothelial scarring and subsequent aneurysm formation [8]. Later histopathological evidence showed active invasion of the vascular wall by viable tumour emboli. Current hypothesis, therefore, assumes that tumour material from a cardiac myxoma embolises into the vasa vasorum of peripheral arteries and subsequently proliferates into the vessel wall, leading to a weakening of subintimal tissue, such as the internal elastic lamina, with subsequent aneurysm formation [7, 10].

The natural history and optimal management of cerebral myxomatous aneurysms is unclear. Treatment with chemotherapy in combination with low-dose radiation has been suggested but further research is warranted [15].

In conclusion, we report on a case of cerebral myxomatous aneurysms 1 year after successful resection of a cardiac myxoma. Although this phenomenon is rare, it is important to be

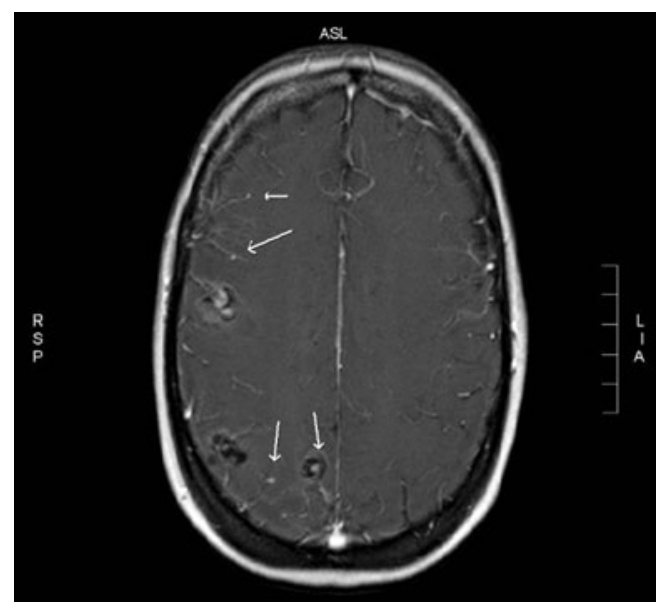

Fig. 2 T2 weighted MR image with gadolinium contrast showing multiple haemorrhagic lesions and (micro) aneurysms (arrows)

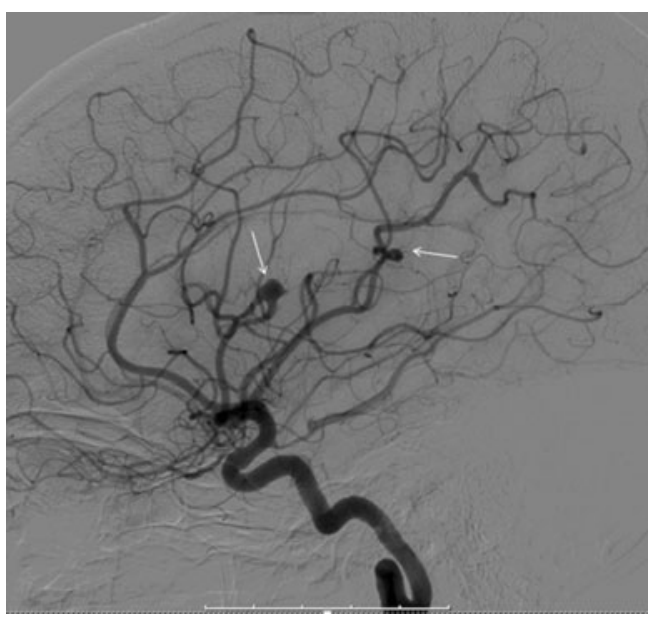

Fig. 3 Cerebral angiography showing aneurysms in the left middle cerebral artery (arrows)

aware of and it must be considered in patients with neurological symptoms even years after successful resection of a myxoma.

Funding None.

Conflict of interests None declared.

Open Access This article is distributed under the terms of the Creative Commons Attribution License which permits any use, distribution, and reproduction in any medium, provided the original author(s) and the source are credited.

\section{References}

1. Van der Zee PM, Van Schuppen J, Van de Brink RBA. Rightsided invasive metastatic thymoma of the heart. Neth Heart J. 2011;19:392-4.

2. Baks T, Galema TW, Bakker J, et al. A rare case of massive papillary fibroelastoma of the right ventricular free wall. Neth Heart J. 2012;20:330-1.

3. Straus R, Merliss R. Primary tumor of the heart. Arch Pathol. 1945;39:74-8.

4. Reynen K. Cardiac myxomas. N Engl J Med. 1995;333:1610-7.

5. DeSousa AL, Muller J, Campbell RL, et al. Atrial myxoma: a review of the neurological complications, metastases and recurrences. J Neurol Neurosurg Psychiatry. 1978;41:1119-24.

6. Liesting C, Ramjankhan FZ, van Herwerden LA, et al. Systemic embolisation as presentation and recurrence of cardiac myxoma two years after surgery. Neth Heart J. 2010;18:499-502.

7. Burton C, Johnston J. Multiple cerebral aneurysms and cardiac myxoma. N Engl J Med. 1970;282:35-65.

8. Stoane L, Allen JH, Collins HA. Radiologic observations in cerebral embolization from left heart myxomas. Radiology. 1966;87:262-6.

9. Chen Z, Wang YL, Ye W, et al. Multiple intracranial aneurysms as delayed complication of atrial myxoma. Interv Neuroradiol. 2005;11:251-4. 
10. Sabolek M, Bachus-Banaschak K, Bachus R, et al. Multiple cerebral aneurysms as delayed complication of left cardiac myxoma: a case report and review. Acta Neurol Scand. 2005;111:345-50.

11. Walker MT, Kilani RK, Toye LR, Bird CR. Central and peripheral fusiform aneurysms six years after left atrial myxoma resection. $\mathrm{J}$ Neurol Neurosurg Psychiatry. 2003;74:281-2.

12. Jean WC, Walski-Easton SM, Nussbaum ES. Multiple intracranial aneurysms as delayed complications of an atrial myxoma: case report. Neurosurgery. 2001;49:200-02.
13. Sedat J, Chau A, Dunac N, et al. Multiple cerebral aneurysms caused by cardiac myxoma. Interv Neuroradiol. 2007;13:179 84.

14. Tamuleviciute E, Taeshineetanakul $\mathrm{P}$, Terbrugge $\mathrm{K}$, et al. Myxomatous aneurysms. Interv Neuroradiol. 2011;17:18894.

15. Bernet F, Stulz PM, Carrel TP. Long term remission after resection, chemotherapy, and irradiation of a metastatic myxoma. Ann Thorac Surg. 1998;66:1791-2. 